\title{
Treatment Patterns Among Patients with Rheumatoid Arthritis in Puerto Rico
}

Eory Madera Miranda · Xue Han · Sang Hee Park · Sonick Suri •

Manasi Suryavanshi

Received: September 15, 2021 / Accepted: November 23, 2021 / Published online: January 24, 2022

(c) The Author(s) 2022

\section{ABSTRACT}

Introduction: Racial and ethnic disparities in rheumatoid arthritis (RA) have been identified in the United States, with higher levels of disease activity and worse functional status reported in Hispanic patients compared with their white counterparts. Although RA is one of the most prevalent health conditions in Puerto Rico, few studies have previously examined the characteristics or treatment patterns of patients receiving biological and targeted synthetic disease-modifying anti-rheumatic drugs (b/ tsDMARDs) in this population.

Methods: This was a retrospective cohort study using data extracted from the Advanced Business Management Organization database, which represents around 70\% of pharmacy claims in Puerto Rico. Patients with RA were included if they had $\geq 1$ prescription for any approved $\mathrm{b} / \mathrm{tsDMARD}$ during the index period

Supplementary Information The online version contains supplementary material available at https:// doi.org/10.1007/s40744-021-00408-7.

E. M. Miranda · X. Han · S. H. Park ·

M. Suryavanshi $(\bowtie)$

Bristol Myers Squibb, 3401 Princeton Pike,

Lawrenceville, NJ 08648, USA

e-mail: manasi.suryavanshi@bms.com

S. Suri

Mu Sigma Inc, No 3400, Dundee Rd, Suite 160,

Northbrook, IL, USA
(January 2016 to July 2018), and $\geq 2$ years of follow-up. The objective was to describe and compare the demographic and clinical characteristics of patients with RA being treated with b/tsDMARD therapy in Puerto Rico, and to evaluate the treatment patterns among these patients.

Results: Most patients (74\%) received tumor necrosis factor inhibitors (TNFis) as index therapy, followed by abatacept (17\%), Janus kinase inhibitors (JAKis; 5\%), and other non-TNFis (4\%). Similar trends were observed in subsequent lines of therapy, although abatacept was more frequently used in these later lines versus index therapy. At 2 years, $62 \%$ of patients had discontinued their index therapy and $17 \%$ had switched to an alternative b/tsDMARD; only $21 \%$ persisted with index therapy. The percentage of patients who were persistent with their index therapy at the end of year 2 was $28 \%$ for JAKis, $36 \%$ for abatacept, $41 \%$ for TNFis, and $45 \%$ for other non-TNFis.

Conclusions: These findings demonstrate that despite the availability of several b/tsDMARDs, patients with RA in Puerto Rico still experience substantial treatment disruption, with almost two-thirds of patients discontinuing their index therapy within 2 years.

Keywords: Biologic disease-modifying antirheumatic drugs; Lines of treatment; Persistence; Pharmacy claims data; Switching; 
Targeted synthetic disease-modifying antirheumatic drugs; Puerto Rico

\section{Key Summary Points}

\section{Why carry out this study?}

Rheumatoid arthritis (RA) is one of the most prevalent health conditions in Puerto Rico, but little is known about the characteristics and patterns of treatment with biological and targeted synthetic disease-modifying anti-rheumatic drugs (b/tsDMARDs) in this population.

Understanding patient characteristics and treatment patterns in real-world populations with RA is important and may contribute to improving patient care.

\section{What was learned from the study?}

Treatment disruption was observed in most patients, with only $21 \%$ persisting with their index b/tsDMARD at 24-month follow-up.

Despite the availability of several treatment options, patients with RA in Puerto Rico still have a substantial unmet need that could potentially be improved by adopting a precision medicine-based approach.

\section{INTRODUCTION}

Rheumatoid arthritis (RA) is a systemic, chronic autoimmune disease involving autoantibody production and subsequent inflammation of the synovial tissue $[1,2]$. Inflammation in the joint tissue and antibody burden resulting from RA leads to increasing loss of joint mobility, erosion of bone in the affected joints, and disability [1, 2].

In the United States (US) alone, RA affects around 1.3 million adults, predominantly women [3]. The goal of RA treatment is clinical remission, defined as no significant signs or symptoms of inflammatory disease activity [4]. The first line of therapy (LoT) is usually methotrexate (MTX), either alone or in combination with other conventional disease-modifying anti-rheumatic drugs (cDMARDs) [5]. If the patient does not respond adequately to cDMARDs they will usually be treated with a biological DMARD (bDMARD), such as a tumor necrosis factor inhibitor (TNFi), T-cell co-stimulation modulator, interleukin-6 (IL-6) inhibitor, or anti-CD20 monoclonal antibody. Alternatively, they may receive a Janus kinase inhibitor (JAKi) or other targeted synthetic DMARD (tsDMARD) [5].

Current US guidelines for RA do not express a preference for TNFi over non-TNFi treatment, or vice versa, recommending instead that the choice of treatment be based on clinical judgement and individual patient preference [6]. In line with this strategy, a recent large US healthcare registry study found no significant difference in efficacy after 1 year of treatment with TNFi versus non-TNFi agents [7].

Although arthritis is one of the most prevalent health conditions in Puerto Rico [8], few studies have been conducted in Puerto Rican or Hispanic populations specifically [9-11]. Studies in the broader US population have identified some racial and ethnic disparities, reporting higher levels of disease activity and worse functional status in Hispanic patients compared with white patients, although it is not clear why this is the case [12-14]. Several studies have found that starting $\mathrm{b} / \mathrm{tsDMARD}$ treatment early in the disease course (within 12 months of initial symptoms) improves long-term outcomes in RA, and this has been confirmed in the Puerto Rican population [10]. However, little is known about the patient characteristics or patterns of treatment among people receiving b/tsDMARD treatment for RA in Puerto Rico. The few studies that have been performed have been small $[9,10]$ or have only included particular subsets of patients (e.g. Medicaid and Medicaid-Medicare dual beneficiaries [11]), and may not be representative of the overall RA population in Puerto Rico.

The aim of this study was to describe and compare the characteristics of a large, representative sample of patients with RA treated 
with b/tsDMARD therapy in Puerto Rico, and to evaluate the treatment patterns among these patients.

\section{METHODS}

\section{Data Source}

This retrospective cohort study used data extracted from the Advanced Business Management Organization (BMO) database, the largest aggregator of pharmacy claims data in Puerto Rico. The database contains transactional data from a group of four specialty pharmacies in Puerto Rico [San Rafael, SPS Specialty Pharmacy, Special Care Pharmacy, and Alivia Health Specialty Pharmacy (formerly Best Option)], representing around $70 \%$ of all pharmacy claims in Puerto Rico. The transactional data are directly related to the prescription received at the pharmacy. International Classification of Diseases, 10th Revision (ICD-10) information is used to identify pharmacy claims for RA and brand information is used to identify the drugs prescribed to the patient. Baseline information can be extracted using index diagnosis month, ICD-10 code for any diagnosis, and brand for any drug check, though patient characteristic information in the dataset is limited. The data extraction process automatically selects the required fields and specific drugs of interest and de-identifies all data; thus, it is fully compliant with US patient confidentiality requirements (the Health Insurance Portability and Accountability Act). This study did not require review or approval by ethics committees, or informed consent. Patient data were deidentified and met the Health Insurance Portability and Accountability Act of 1996 requirements to ensure patient anonymity.

\section{Study Design and Population}

The study period was January 1, 2016 to July 31, 2020 (Supplemental Fig. 1). Patients treated with a b/tsDMARD between January 1, 2016 and July 31, 2018 were identified. The Advanced BMO database only provides data on the month of drug prescription, not the day; thus, the first day of the month of the first b/tsDMARD prescription after an RA diagnosis was defined as the index date. Patients were followed from the index date to the end of the study period, or the date of the last prescription (for any medication) recorded in the database.

Patients were included if they had at least one RA diagnosis during the study period, as identified using ICD-10-CM codes (Supplemental Table 1), at least one prescription for any b/tsDMARD approved for RA in Puerto Rico on or after the first RA diagnosis and during the index period (January 2016 to July 2018), and at least 2 years of follow-up. Follow-up duration was calculated as last prescription in the database (for any medication) index date +1 . Patients who had multiple prescriptions for $\mathrm{b} / \mathrm{tsDMARDs}$ in the index month were excluded.

The study cohort was divided into four mutually exclusive treatment subgroups according to the index b/tsDMARD: (1) abatacept, (2) TNFi (adalimumab, certolizumab, etanercept, golimumab, or infliximab), (3) JAKi (baricitinib, tofacitinib, or upadacitinib), and (4) other bDMARDs (anakinra, rituximab, sarilumab, or tocilizumab), referred to hereafter as 'non-TNFi'. Where available, biosimilars were grouped with the original drug (e.g., infliximab and biosimilar infliximab were counted together).

\section{Outcomes and Measures}

The primary outcome variables described for the four index treatment subgroups were patient characteristics and treatment patterns. Patient characteristics included health plan type (Medicare, Medicaid, or commercial), patient region (North, South, East, or West Puerto Rico), and prescriber specialty (family/general practitioner, internal medicine, rheumatology, or other). Treatment pattern outcomes included rates of initiation, switching, persistence, and discontinuation at each LoT. Mean time to switching and discontinuation and duration of treatment persistence were also assessed. 
As it is not possible to determine whether a patient's first prescription in the Advanced BMO database is their first lifetime prescription, LoT was defined as follows: 'early line' (EL), representing the first index b/tsDMARD prescription filled during the study period; 'subsequent line' $(\mathrm{SqL})$, representing the second $\mathrm{b} /$ tsDMARD prescription filled during the study period; and 'later line' (LL), representing the third $b /$ tsDMARD prescription filled.

A treatment switch was defined as a pharmacy record for $a \mathrm{~b} / \mathrm{tsDMARD}$ that was different to the index $b /$ tsDMARD drug within the time period of two times the 'days of supply' from the end of the period covered by the previous prescription. Days of supply was imputed using the recommended doses for drugs wherever required. Discontinuation of the index drug was defined as a period of at least two times the days of supply from last prescription fill with no pharmacy record for the index b/tsDMARD. The patient was considered to have discontinued in the month at the end of the period covered by the last prescription.

Patients who continued their treatment during the study period (without treatment switching or discontinuation) were defined as persistent. Persistence was calculated using the gap of two times the days of supply from the end of the period covered by the last prescription fill. For patients who discontinued treatment, persistence duration was capped to the date of discontinuation of the index $\mathrm{b} / \mathrm{tsDMARD}$. For patients who switched treatment, persistence was calculated up to the start date of the next LoT.

\section{Statistical Analyses}

Descriptive statistics were used for all study objectives. The following descriptive statistics were used to summarize patient characteristics, overall and by treatment subgroup: for categorical variables, counts and percentages, and for continuous variables, means, standard deviations, and medians, as appropriate. Statistical comparisons were performed across treatment cohorts using Chi-square tests and Fisher's exact test with Monte Carlo simulation, as appropriate.

For treatment patterns, the top five treatment patterns at a drug level up to the third LoT were described. Within each LoT, the number and percentage of patients who switched, discontinued, and were persistent were determined and described by treatment cohort. Duration on each LoT and mean time to switching or discontinuation were determined for each index drug and index drug cohort.

\section{RESULTS}

\section{Patient Characteristics}

In the Advanced BMO database, 2077 patients met the eligibility criteria (Table 1). Index $\mathrm{b} /$ tsDMARD prescriptions were primarily from rheumatologists $(65 \%) ; 11 \%$ were issued by internal medicine specialists and $6 \%$ by family/general practitioners. Median (standard deviation) follow-up was 1188 (313.9) days. The majority of patients were Medicaid/Medicare beneficiaries (74\%), while $25 \%$ were on a commercial health plan type. A significant association was identified between the patients' health plan type and the treatment they received (Chisquare test $p$ value $<0.0001$ ).

\section{Treatment Distribution}

The majority of patients received TNFi (74\%), followed by abatacept (17\%), JAKi (5\%), and other non-TNFi (4\%) treatments as their index/ EL treatment (Supplemental Table 2; Fig. 1A). Adalimumab was the most frequently prescribed TNFi (41\%), followed by etanercept (23\%), golimumab (6\%), infliximab $(2 \%)$, and certolizumab (1\%). Tofacitinib was the only JAKi used as index/EL treatment. The most frequently used non-TNFi was tocilizumab (4\%) followed by rituximab (1\%); only one patient received sarilumab as their index/EL treatment.

Among the 580 patients who received SqL therapy, a majority received TNFi (40\%), closely followed by abatacept (37\%) (Fig. 1B). Adalimumab was once again the most frequently 
Table 1 Patient demographics and baseline characteristics by treatment subgroup

\begin{tabular}{|c|c|c|c|c|c|c|c|c|c|}
\hline & \multicolumn{2}{|c|}{$\mathbf{A B A}$} & \multicolumn{2}{|l|}{ TNFi } & \multicolumn{2}{|c|}{ Non-TNFi } & \multicolumn{2}{|c|}{ JAKi } & \multirow[t]{2}{*}{$p$ value $^{\mathrm{a}}$} \\
\hline & $\bar{N}$ & $\%$ & $N$ & $\%$ & $\bar{N}$ & $\%$ & $\bar{N}$ & $\%$ & \\
\hline Patient count & 360 & 100 & 1535 & 100 & 86 & 100 & 96 & 100 & \\
\hline \multicolumn{10}{|l|}{ Health plan type } \\
\hline Commercial & 78 & 21.67 & 388 & 25.28 & 20 & 23.26 & 39 & 40.63 & \multirow[t]{5}{*}{$<0.0001$} \\
\hline Medicaid & 89 & 24.72 & 482 & 31.40 & 14 & 16.28 & 9 & 9.38 & \\
\hline Medicare & 191 & 53.06 & 652 & 42.48 & 50 & 58.14 & 48 & 50.00 & \\
\hline Others/multiple & 2 & 0.56 & 13 & 0.85 & 2 & 2.33 & 0 & 0.00 & \\
\hline Missing & 0 & 0.00 & 0 & 0.00 & 0 & 0.00 & 0 & 0.00 & \\
\hline \multicolumn{10}{|l|}{ Patient region } \\
\hline North & 230 & 63.89 & 982 & 63.97 & 60 & 69.77 & 71 & 73.96 & \multirow[t]{5}{*}{0.1615} \\
\hline South & 18 & 5.00 & 109 & 7.10 & 3 & 3.49 & 4 & 4.17 & \\
\hline East & 53 & 14.72 & 239 & 15.57 & 8 & 9.30 & 9 & 9.38 & \\
\hline West & 58 & 16.11 & 197 & 12.83 & 14 & 16.28 & 12 & 12.50 & \\
\hline Missing & 1 & 0.28 & 8 & 0.52 & 1 & 1.16 & 0 & 0.00 & \\
\hline \multicolumn{10}{|l|}{ Prescriber specialty } \\
\hline Family/general practice & 19 & 5.28 & 111 & 7.23 & 2 & 2.33 & 1 & 1.04 & \multirow[t]{5}{*}{0.0272} \\
\hline Internal medicine & 37 & 10.28 & 186 & 12.12 & 9 & 10.47 & 5 & 5.21 & \\
\hline Rheumatology & 246 & 68.33 & 960 & 62.54 & 59 & 68.60 & 75 & 78.13 & \\
\hline Others/multiple & 58 & 16.11 & 275 & 17.92 & 16 & 18.60 & 15 & 15.63 & \\
\hline Missing & 0 & 0.00 & 3 & 0.20 & 0 & 0.00 & 0 & 0.00 & \\
\hline
\end{tabular}

$A B A$ abatacept; JAKi Janus kinase inhibitor; TNFi tumor necrosis factor inhibitor

${ }^{a}$ Chi-square test was used to compute $p$ values for health plan type, patient region, and specialty type. Fisher's exact test with Monte Carlo simulation was used to estimate $p$ values for health plan

prescribed TNFi (17\%), followed by etanercept (14\%); proportions of patients receiving golimumab, infliximab, and certolizumab were similar to those observed in EL treatment $(6,3$, and $1 \%$, respectively). Higher proportions of patients received JAKi (12\%), and other nonTNFi (11\%) treatments as SqL therapy compared with EL therapy. Tofacitinib was the most frequently prescribed JAKi (11\%).

Among the 205 patients who received LL therapy, TNFi were once again the most frequently prescribed drug group (48\%), followed by abatacept (21\%) (Fig. 1C). A majority of patients who received TNFi were treated with etanercept (20\%); adalimumab was prescribed in $12 \%$, golimumab in $10 \%$, infliximab in $4 \%$, and certolizumab in $2 \%$ of patients. Higher proportions of patients received JAKi (16\%) and other non-TNFi (16\%) treatments as LL treatment compared with EL and SqL treatment. Tofacitinib was the most frequently prescribed JAKi (11\%); higher proportions of patients were treated with upadacitinib (3\%) and baricitinib (2\%) compared with earlier lines of treatment.

Treatment rates for non-TNFi in SqL and LL were similar to that observed in EL. No patient received anakinra in any line of treatment. 


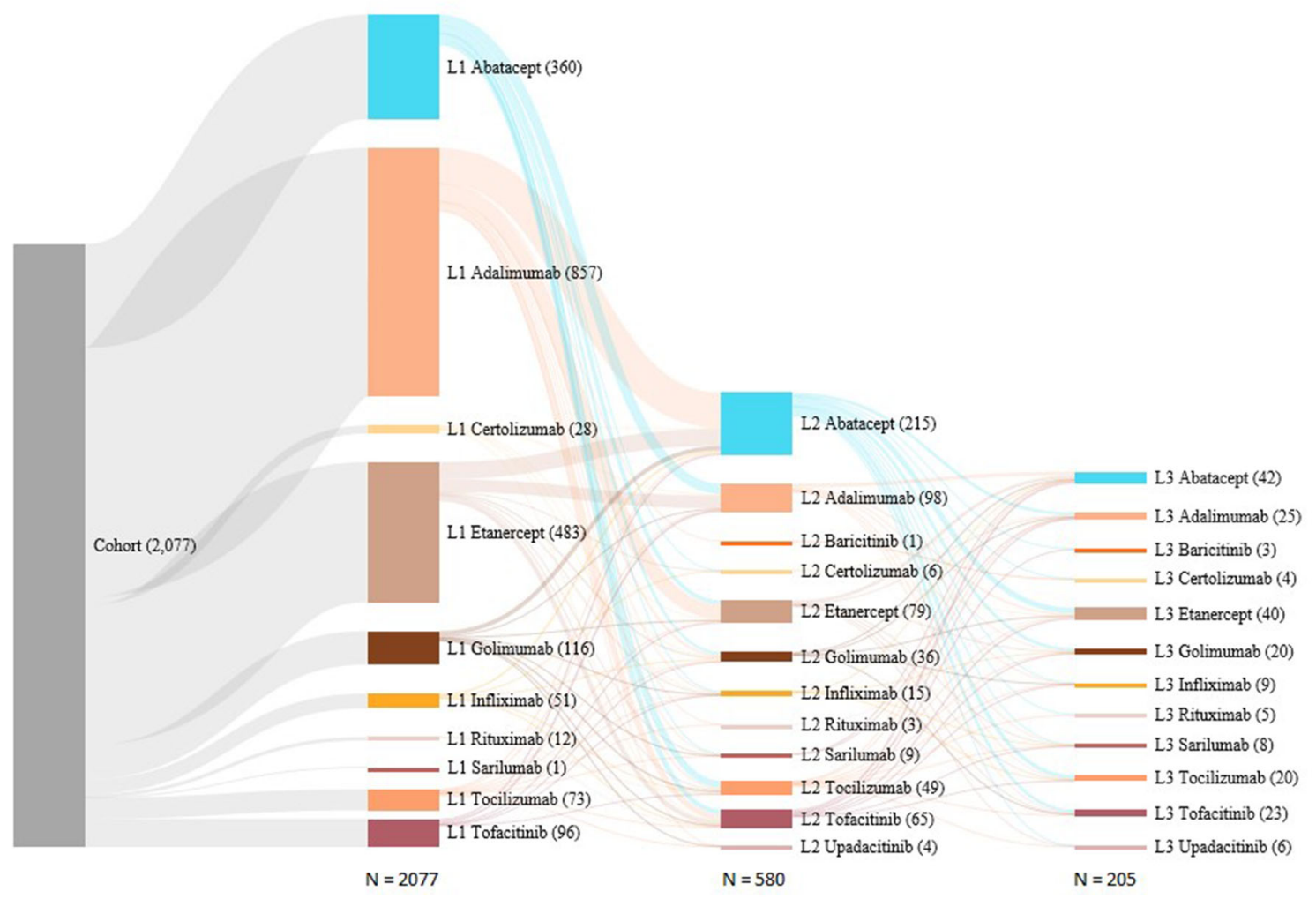

Fig. 1 Treatment patterns over 24 months. L1: Early-line treatment; L2: Subsequent-line treatment; L3: Later-line treatment

\section{Treatment Patterns}

The majority of patients (62\%) discontinued index/EL therapy over the 24-month follow-up period, regardless of therapy; $21 \%$ were persistent and $17 \%$ switched treatment; $51 \%$ also discontinued SqL treatment during follow-up; $26 \%$ were persistent and $23 \%$ switched treatment. Overall, $10 \%$ of patients who discontinued EL therapy did not reinitiate or start a new b/tsDMARD during follow-up and were identified as pure discontinuers; $46 \%$ and $10 \%$ of patients reinitiated the same treatment after discontinuing EL and SqL therapy, respectively.

Overall, the median duration of treatment persistence for medications prescribed as index/ EL treatment was 536 days [interquartile range (IQR): 210-1001 days]. Median duration of persistence was 574 days for non-TNFi (IQR: 29-1025 days), 539 days for TNFi (IQR: 136-10,906 days), 457 days for abatacept (IQR: 201-937 days), and 275 days for JAKi (IQR:
122-791) (Fig. 2A). For the 73 patients who received tocilizumab as index/EL treatment, median duration of persistence was 737 days (IQR: 271-1025 days); the patient who received sarilumab had a median persistence of 823 days. Median (IQR) duration of TNFi persistence was 695 (307-1037) days for infliximab, 606 (244-1096) days for adalimumab, 487 (181-974) days for etanercept, 480 (163-846) days for golimumab, and 426 (136-895) days for certolizumab.

More than half of all patients persisted with EL treatment at the end of year 1 in the nonTNFi (63\%), TNFi (62\%), and abatacept (58\%) subgroups (Fig. 2B). Of the 96 patients treated with EL tofacitinib, the only JAKi prescribed at this stage, $45 \%$ persisted with treatment at the end of year 1 . In the non-TNFi group, 50 patients (68\%) who received EL tocilizumab persisted with treatment at the end of year 1 ; the patient who received EL sarilumab continued with treatment into year 2 , as did three of 
(A)

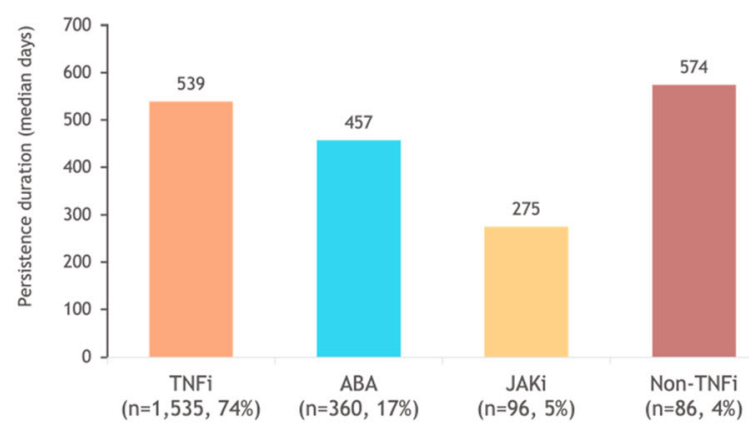

Fig. 2 Treatment persistence. A Median duration of persistence for index/EL treatment; $\mathbf{B}$ persistence by year ${ }^{1}$ of follow-up. ${ }^{1}$ Year 1 persistence is $\%$ of patients who were persistent for $\geq 365$ days; year 2 persistence is $\%$ of patients who were persistent for $\geq 730$ days. Percentages

the 12 patients (25\%) who received EL rituximab. Proportions of patients persisting with TNFi treatment at the end of year 1 were $71 \%$ for infliximab, $65 \%$ for adalimumab, $61 \%$ for certolizumab, $59 \%$ for golimumab, and $56 \%$ for etanercept.

A similar pattern of treatment persistence was observed across the treatment subgroups at the end of year 2. The percentage of patients who were persistent with their index therapy at the end of year 2 was $28 \%$ for JAKis, $36 \%$ for abatacept, $41 \%$ for TNFis, and $45 \%$ for other non-TNFis.

\section{DISCUSSION}

Although multiple therapeutic options are now available for RA, not all patients receive guideline-recommended therapies [15] and attainment of remission or low disease activity remains challenging for many patients [16]. Understanding patient characteristics and treatment patterns in real-world populations with RA is important and may provide information that can contribute to improving patient care [17-21]. This large real-world study provides insights into the characteristics and treatment patterns among patients with RA in Puerto Rico, highlighting key areas of unmet need in this little-studied population. We found that the majority of patients (62\%)

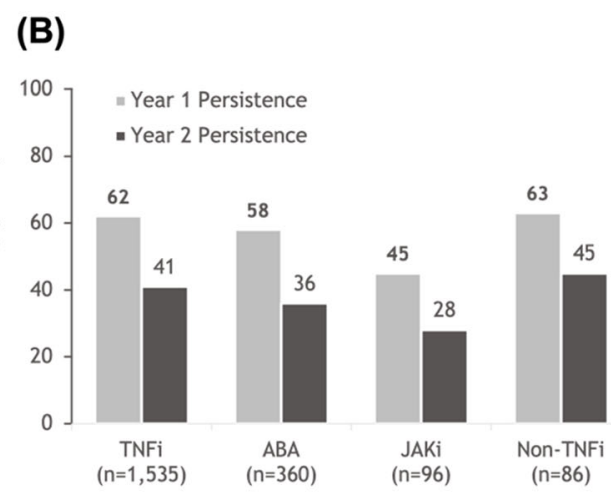

are calculated from the overall number of patients receiving early-line treatment in each drug group. $A B A$ abatacept, $J A K i$ Janus kinase inhibitor, TNFi tumor necrosis factor inhibitor

discontinued their index/EL therapy during the study period, regardless of the type of therapy they were prescribed. Rates of switching were also high (17\%), leaving only $21 \%$ of patients persistent with EL therapy at 24-month followup.

To the best of our knowledge, this is the first Advanced BMO database study to examine the characteristics and treatment patterns of patients receiving $b / t s D M A R D S$ for $R A$ in a large, representative group of patients in Puerto Rico. In our study, $74 \%$ of the population received TNFis, followed by $17 \%$ abatacept, 5\% JAKi, and 4\% non-TNFi treatments. Abatacept treatment was slightly more common in SqL and LL treatment: 17,37 , and $20 \%$ of patients received abatacept as EL, SqL, and LL therapy, respectively. This treatment distribution is similar to what has been reported previously in the wider US population, where TNFis are also the most commonly used bDMARDs for the treatment of RA [6, 11].

Previous studies have provided evidence of disparities in RA treatment in the Puerto Rican population $[9,10]$. A cross-sectional study of Puerto Ricans with RA looked at disparities between those receiving their healthcare in a managed care system $(n=67)$ and non-indigent patients treated in fee-for-service settings $(n=147)$ [10]. Patients in the managed care setting had more joint deformities, extra-articular manifestations, arterial hypertension, type 
2 diabetes mellitus, cardiovascular events, fibromyalgia syndrome, and poorer functional status. These patients also had lower exposure to bDMARDs than those treated in fee-for-service settings. A report on the distribution of DMARDs prescribed to Medicaid and Medicaid-Medicare dual beneficiaries of a federally funded health insurance program in Puerto Rico found that 24,16 , and $7 \%$ of prescriptions for RA during 2016 were for adalimumab, etanercept, and abatacept, respectively [11]. The use of newer b/tsDMARDs such as abatacept, golimumab, tocilizumab, tofacitinib, and certolizumab accounted for approximately $28 \%$ of all b/tsDMARD prescriptions dispensed to RA patients during the study period.

In our study, significant associations were identified between the Puerto Rico RA patients' health plan type and the treatment they received. Although TNFis were by far the most common treatment received across health plan types, a larger proportion of patients with commercial or Medicare health plans received JAKi and other non-TNFi treatments than those with Medicaid plans, and more than $50 \%$ of patients receiving EL abatacept treatment had Medicare coverage. This finding suggests that the type of health plan a patient has may dictate their access to different therapies, at least to some extent.

In terms of treatment patterns, most patients discontinued their EL treatment over the 24-month follow-up period regardless of therapy: tofacitinib $(76 \%)$, abatacept $(63 \%)$, TNFi (62\%), and non-TNFi (52\%). Notably, only 9\% and $14 \%$ of patients on EL and SqL abatacept treatment, respectively, discontinued over the 24-month follow-up period. The comparable efficacy of abatacept with TNFi and other nonTNFi has previously been established in a large network meta-analysis of clinical trial data in the pre-JAKi era [22] and in real-world observational studies [23, 24].

Similar trends in persistence duration (time to discontinuation or switching) were observed during EL and SqL therapy. Tocilizumab was associated with the longest observed EL treatment persistence among treatments prescribed to $>1$ patient (median 737 days); the one patient who received EL sarilumab had a treatment persistence of 823 days. Overall, 58\% of patients treated with EL abatacept and $45 \%$ of patients treated with tofacitinib were persistent at the end of 1 year.

Overall, the results indicate that despite the availability of numerous treatment options for RA in Puerto Rico, patients typically cycle through many treatments over the course of their lives, with high rates of discontinuation, switching to alternative therapies, and reinitiation of the original therapy observed over a 2 -year period. Our results are in line with a previous report, based on US Medicare data, that only one in 20 patients remain on their first bDMARD and that nearly $25 \%$ of disruptions occur within 60 days of initiating treatment, over $50 \%$ within 1 year, and over $90 \%$ by year 3 [25].

The high level of observed treatment disruption in our study is important because undertreatment of RA may lead to insufficient control of inflammation, leading to further disease progression. The results indicate that despite the availability of several treatment options, patients with RA in Puerto Rico still have a substantial unmet need. Often the choice of first/EL treatment is not based on an optimal strategy for controlling inflammation, but rather a trial-and-error approach that, when it fails, can impact on subsequent treatment efficacy. Adopting a precision medicine-based approach in Puerto Rico could potentially improve persistence with the first/EL treatment and lead to better patient outcomes. Further studies using other databases would be welcomed to expand on the current results and to provide more information about treatment patterns and persistence among patients with RA in Puerto Rico.

\section{Study Limitations}

A strength of this study is that it takes an innovative approach, utilizing a database that has not previously been used for this type of research. The Advanced BMO database is the largest aggregator of pharmacy claims data in Puerto Rico, so is the most useful data source for examining treatment patterns in the country; 
however, it has some limitations. Patient demographic and clinical information in the dataset is limited. The database only provides information on drug prescription by month not by day: if a patient has multiple drug prescriptions in the same month, it is not possible to identify the treatment sequence. To utilize the data despite this limitation, patients with multiple prescriptions for $\mathrm{b} / \mathrm{tsDMARDs}$ in the index month were excluded. The fact that drug prescriptions are only available by month also means that time to discontinuation and time to switching calculations are within a margin of error of approximately 31 days. The index prescription is the first available prescription in the database so does not necessarily represent the patient's first lifetime prescription. Thus, we designated the first available prescription for each patient their 'early line' of therapy, followed by their 'subsequent line' and 'later line' where applicable. Continuous enrollment information is unavailable in the database; thus, the study required patients to have $\geq 1$ pharmacy claim during segments of the 24-month follow-up. Owing to variations in drug rebate agreements, this may have led to smaller sample sizes in some treatment groups, particularly the TNFi group.

Our study focused on biologic and newer generation targeted synthetic DMARDS. It is possible that patients were taking conventional DMARDs or other therapies not accounted for by our study design, and this may have influenced the low persistence rates. In addition, the association between health plan type and treatment type noted in our study raises questions about the insurance guarantee contents of different health plans; however, this information was not available and thus we cannot be sure whether the specifics of insurance policies and/or coverage influenced treatment selection. The study is also subject to the inherent limitations of any claims database study, in that miscoding of diagnoses and/or treatments cannot be excluded, and we cannot be sure that patients actually took the drugs as prescribed. Finally, the study is only descriptive in nature, and as the baseline demographic and clinical characteristic information in the dataset is limited, the results of this study should be interpreted considering potential selection bias; thus, no causal relationships can be inferred from the results.

\section{CONCLUSIONS}

This large retrospective, real-world study provides insights into the characteristics and treatment patterns among patients with RA in Puerto Rico. Most patients received TNFi agents as their index/EL treatment. Similar trends were observed for SqL and LL treatment, though use of abatacept, JAKis, and non-TNFis was more common in these lines compared with EL treatment. The majority of patients $(62 \%)$ discontinued their EL therapy over time, regardless of the type of therapy they were prescribed. A further $17 \%$ of patients switched treatments, leaving only $21 \%$ of patients persistent at 2 years. These findings demonstrate that patients with RA in Puerto Rico still have a substantial unmet need. A precision medicinebased approach to first-line treatment could have the potential to improve persistence and patient outcomes.

\section{ACKNOWLEDGEMENTS}

Funding. This study and journal's Rapid Service Fee was supported by Bristol Myers Squibb.

Medical Writing, Editorial, and Other Assistance. Professional medical writing assistance was provided by LATITUDE (powered by AXON Communications) and was funded by Bristol Myers Squibb.

Authorship. All named authors meet the International Committee of Medical Journal Editors (ICMJE) criteria for authorship for this article, take responsibility for the integrity of the work as a whole, and have given their approval for this version to be published.

Author Contributions. All authors contributed to and approved the manuscript. Eory 
Madera Miranda: conceptualization, resources, visualization, validation, investigation, supervision, writing, and project administration. Xue Han: validation, review, and editing. Sang Hee Park: validation, review, and editing. Sonick Suri: methodology, formal analysis, and data curation. Manasi Suryavanshi: conceptualization, resources, visualization, validation, investigation, methodology, formal analysis, data curation, review, and editing.

Disclosures. Eory Madera Miranda owns stock in BMS, at the time of the research was an employee of BMS, and is currently employed by Oncopeptides, Inc; Xue Han was an employee of BMS at the time of research and is now an employee of Otsuka Pharmaceutical \& Commercialization Inc; Sang Hee Park owns stock in BMS; Sonick Suri has nothing to disclose; Manasi Suryavanshi owns stock in BMS.

Compliance with Ethics Guidelines. This study did not require review or approval by ethics committees, or informed consent. Patient data were de-identified and met the Health Insurance Portability and Accountability Act of 1996 requirements to ensure patient anonymity.

Data Availability. The data that support the findings of this study are available from Advance BMO, LLC but restrictions apply to the availability of these data, which were used under license for BMS, and so are not publicly available. Data are, however, available from the authors upon reasonable request and with permission of Advance BMO, LLC.

Open Access. This article is licensed under a Creative Commons Attribution-NonCommercial 4.0 International License, which permits any non-commercial use, sharing, adaptation, distribution and reproduction in any medium or format, as long as you give appropriate credit to the original author(s) and the source, provide a link to the Creative Commons licence, and indicate if changes were made. The images or other third party material in this article are included in the article's Creative Commons licence, unless indicated otherwise in a credit line to the material. If material is not included in the article's Creative Commons licence and your intended use is not permitted by statutory regulation or exceeds the permitted use, you will need to obtain permission directly from the copyright holder. To view a copy of this licence, visit http://creativecommons.org/licenses/by$\mathrm{nc} / 4.0 /$.

\section{REFERENCES}

1. McInnes IB, Schett G, et al. The pathogenesis of rheumatoid arthritis. N Engl J Med. 2011;365(23): 2205-19.

2. Chaudhari K, Rizvi S, Syed BA, et al. Rheumatoid arthritis: current and future trends. Nat Rev Drug Discov. 2016;15(5):305-6.

3. Crane M, Juneja M, Allen J, et al. Epidemiology and treatment of new-onset and established rheumatoid arthritis in an insured US population. Arthritis Care Res. 2015;67(12):1646-55.

4. Smolen JS, Breedveld FC, Burmester GR, et al. Treating rheumatoid arthritis to target: 2014 update of the recommendations of an international task force. Ann Rheum Dis. 2016;75(1):3-15.

5. Rein P, Mueller RB. Treatment with biologicals in rheumatoid arthritis: an overview. Rheumatol Ther. 2017;4(2):247-61.

6. Fraenkel L, Bathon JM, England BR, et al. American college of rheumatology guideline for the treatment of rheumatoid arthritis. Arthritis Rheumatol. 2021; 73(7):1108-23. https://doi.org/10.1002/art.41752.

7. Pappas DA, St. John G, Etzel CJ, et al. Comparative effectiveness of first-line tumour necrosis factor inhibitor versus non-tumour necrosis factor inhibitor biologics and targeted synthetic agents in patients with rheumatoid arthritis: results from a large US registry study. Ann Rheum Dis. 2021;80(1): 96-102.

8. Mattei J, Tamez M, Rios-Bedoya CF, et al. Health conditions and lifestyle risk factors of adults living in Puerto Rico: a cross-sectional study. BMC Public Health. 2018;18:491.

9. Varela-Rosario N, Arroyo-Avila M, Fred-Jiménez $\mathrm{RM}$, et al. Long-term outcomes in Puerto Ricans with rheumatoid arthritis receiving early treatment with disease-modifying anti-rheumatic drugs using the American College of Rheumatology Definition of Early RA. Open Rheumatol J. 2017;11:136-44. 
10. Santiago-Casas Y, Gonzalez-Rivera T, Castro-Santana L, et al. Impact of managed care health insurance system for indigent patients with rheumatoid arthritis in Puerto Rico. Clin Rheumatol. 2013;32: 763-9.

11. Hernández-Muñoz JJ, Wei W, Sierra-Zorita R. Prevalence of rheumatoid arthritis and drug dispensing patterns among Medicaid and Medicaid-Medicare dually eligible in Puerto Rico. Arthritis Care Res (Hoboken). 2021;73(2):199-206.

12. Greenberg JD, Spruill T, Shan Y, et al. Racial and ethnic disparities in disease activity in patients with rheumatoid arthritis. Am J Med. 2013;126(12): 1089-98.

13. Del Rincon I, Battafarano DF, Arroyo RA, Murphy FT, Fischbach M, Escalante A. Ethnic variation in the clinical manifestations of rheumatoid arthritis: role of HLA-DRB1 alleles. Arthritis Rheum. 2003;49:200-8.

14. Karpouzas GA, Dolatabadi S, Moran R, Li N, Nicassio PM, Weisman MH. Correlates and predictors of disability in vulnerable US Hispanics with rheumatoid arthritis. Arthritis Care Res (Hoboken). 2012;64:1274-81.

15. Kern DM, Chang L, Sonawane K, et al. Treatment patterns of newly diagnosed rheumatoid arthritis patients from a commercially insured population. Rheumatol Ther. 2018;5(2):355-69.

16. Pavelka K, Kavanaugh AF, Rubbert-Roth A, Ferraccioli G. Optimizing outcomes in rheumatoid arthritis patients with inadequate responses to disease-modifying anti-rheumatic drugs. Rheumatology (Oxford). 2012;51(Suppl 5):v12-21.

17. Agarwal SK. Core management principles in rheumatoid arthritis to help guide managed care professionals. J Manag Care Pharm. 2011;17:S03-8.
18. Agarwal SK. Biologic agents in rheumatoid arthritis: an update for managed care professionals. J Manag Care Pharm. 2011;17:S14-8.

19. Birnbaum H, Pike C, Kaufman R, Marynchenko M, Kidolezi Y, Cifaldi M. Societal cost of rheumatoid arthritis patients in the US. Curr Med Res Opin. 2010;26:77-90.

20. Ward MM, Javitz HS, Yelin EH. The direct cost of rheumatoid arthritis. Value Health. 2000;3:243-52.

21. Ward MM, Lubeck D, Leigh JP. Long-term health outcomes of patients with rheumatoid arthritis treated in managed care and fee-for-service practice settings. J Rheumatol. 1998;25:641-9.

22. Guyot P, Taylor P, Christensen R, et al. Abatacept with methotrexate versus other biologic agents in treatment of patients with active rheumatoid arthritis despite methotrexate: a network metaanalysis. Arthritis Res Ther. 2011;13(6):R204.

23. Gottenberg JE, Ravaud P, Cantagrel A, et al. Positivity for anti-cyclic citrullinated peptide is associated with a better response to abatacept: data from the 'Orencia and Rheumatoid Arthritis' registry. Ann Rheum Dis. 2012;71(11):1815-9.

24. Lahaye C, Soubrier M, Mulliez A, et al. Effectiveness and safety of abatacept in elderly patients with rheumatoid arthritis enrolled in the French Society of Rheumatology's ORA registry. Rheumatology. 2016;55(5):874-82.

25. Dore R, Chang L, Ji Y, et al. Treatment patterns and persistency following the first biologic DMARD in patients with rheumatoid arthritis: real-world analysis of 2012-2016 US Medicare Data. 2019 ACR/ARP Annual Meeting, Abstract Number 953. 